\title{
Exploring Topic-Based Sharing Mechanisms
}

\author{
Manya Sleeper, Lorrie Faith Cranor, Sarah K. Pearman
}

\author{
Carnegie Mellon University \\ Pittsburgh, PA \\ \{msleeper, lorrie, spearman\}@cmu.edu
}

\begin{abstract}
General-purpose content-sharing platforms make it difficult for users to limit sharing to people interested in particular topics. Additional topic-based controls may allow users to better reach desired audiences. Designing such tools requires understanding current interest-based targeting techniques and the potential impact of additional mechanisms. We present an exploratory, interview-based study $(n=16)$ that addresses these dynamics for Facebook. We use diary-driven probes to explore general topic-based sharing across applications. We then use Facebook-based mockups to probe use cases and design tensions around adding topic-based sharing mechanisms to Facebook. We find that participants currently draw on various audience-limiting and reaching strategies to target interest-based audiences. Participants felt additional topicbased sharing mechanisms on Facebook might allow them to avoid oversharing or offending others and allow them to target improved audiences or share improved content. Usable topic-based sharing tools would also need to account, however, for participants' varied desired engagement strategies.
\end{abstract}

\section{Author Keywords}

selective sharing; access control; topics; targeting strategies; topic-based sharing; Facebook

\section{ACM Classification Keywords}

H.5.m. Information Interfaces and Presentation (e.g. HCI): Miscellaneous

\section{INTRODUCTION}

People sometimes want to share content related to particular topics with others interested in those topics (topic-based sharing). For example, someone might want to share content related to dogs with other dog lovers or content related to sports with other fans of a team. Some services and applications specifically provide different types of topic-based sharing or filtering. For example, Twitter allows users to add hashtags; photo-sharing and blogging sites also often allow

Permission to make digital or hard copies of part or all of this work for personal or classroom use is granted without fee provided that copies are not made or distributed for profit or commercial advantage and that copies bear this notice and the full citation on the first page. Copyrights for third-party components of this work must be honored. For all other uses, contact the Owner/Author.

Copyright is held by the owner/author(s)

CHI 2017, May 06-11, 2017 Denver, CO, USA

ACM 978-1-4503-4655-9/17/05

http: //dx . doi .org/10.1145/3025453 . 3025840 users to tag content. People can join mailing lists on specific topics or participate in discussions on topic-specific forums.

People may also share using services that provide more general-use functionalities. These service choices may be driven by a desire to perform tasks or reach particular audiences $[34,44]$. When these services lack mechanisms that allow users to easily target audiences interested in particular topics, however, users may share in a suboptimal manner.

Facebook, for example, provides access to a variety of audiences, including friends and family, and allows users to perform a wide variety of activities, ranging from photo sharing or conversation to promoting oneself or events [13, 39, 31, 34]. However, Facebook offers limited mechanisms for topic-driven targeting or filtering. This gap has been found to prompt Facebook users to self censor content they might otherwise have wanted to share with audiences present on Facebook or to share content more broadly than they may have desired $[33,22]$.

In this paper we focus on how providing additional topicbased sharing mechanisms on Facebook may allow users to better target desired audiences. Designing these types of tools requires understanding topic-based sharing needs, as well as the use cases and design tensions that introducing such mechanisms to Facebook might create. We take an exploratory approach to this space by addressing 1) how participants currently target content along topic-based dimensions, across services; 2) use cases for specific types of potential topicbased sharing mechanisms on Facebook; and 3) possible design tensions inherent to introducing topic-based sharing to Facebook.

We use a semi-structured interview approach centered around a retrospective diary to probe how participants $(n=16)$ currently share about topics across services. We then focus on Facebook, as an example of a broad social-media platform, to explore potential use cases for topic-based sharing. We probe participants' perceptions of use cases for general, hypothetical topic-based sharing. We then further probe perceived use cases and tensions through walkthroughs of mockups for three potential topic-based sharing mechanisms, centered around the ability to: 1) opt out of topics; 2) opt in to topics; and 3) share on topics without identifying oneself.

We find that participants currently intend content for audiences on different services based on a variety of topic-driven dimensions and may draw on broad targeting techniques to 
try to reach these audiences. Participants described varied use cases for additional topic-based mechanisms on Facebook that might allow them to more narrowly target content using Facebook to avoid the risk of oversharing or offending others on Facebook or other services. Participants felt that the ability to target more interested audiences might also increase the quantity or quality of the content shared or facilitate better discussions. However, adding topic-based sharing to Facebook would not meet all of participants' sharing needs, and designing usable topic-based sharing mechanisms would require consideration of possible design tensions as well as participants' potential engagement strategies.

\section{BACKGROUND AND RELATED WORK}

People may draw on different types of sharing mechanisms to reach desired audiences, ranging from location to time of posting $[41,37,22]$. We focus on the desire to target content based on audience members' interest in the topic of the content. For example one might only want to share pictures of cats with others interested in cats. We explore general uses of topic-based sharing as well as the use cases and tensions around potentially adding three common mechanisms for topic-based sharing to Facebook: the ability to tag content with a topic and allow others to opt out of viewing it, the ability to tag content with a topic and allow others to opt in to viewing it, and the ability to share on a topic without identifying oneself.

\section{Sharing on Facebook}

We use Facebook to explore how adding topic-driven mechanisms to a general-use platform can provide insights into uses and tensions inherent in adding more niche functionality to a general-use site. Facebook is a broad-reaching social media platform. People use it to target a variety of audiences. It also provides diverse functionalities used for a wide variety of activities including photo sharing, discussing politics, receiving or providing resources or social support, collaborating on projects, promoting oneself or others, and archiving content $[13,39,31,34]$.

Because Facebook allows users to combine different tasks and audiences on a single platform, targeting desired audiences can be challenging. Facebook's model can create a dynamic of "context collapse" by forcing users to share content simultaneously across groups that might typically be kept separate offline $[25,20]$. Facebook users may also misperceive their audiences or share with an "imagined audience" that doesn't match their full broadcast context [1, 3, 21].

This dynamic can lead to regret, if content is accidentally broadcast too widely or to unexpected audiences [40]. Social media users, including those on Facebook, must also account for a number of social risks or norms when sharing. They risk being unfriended or unfollowed if others feel that their posts are uninteresting, inappropriate, overly personal, or too frequent [26, 32]. More generally, users can face the risk of "stigma" or the potential for their posts to prompt audience members to perceive them unfavorably [29].

We focus on understanding how current sharing challenges may vary for topic-driven dimensions. We explore how adding topic-based sharing mechanisms may help users address known targeting challenges and sharing risks.

\section{Audience-targeting techniques}

People use a variety of techniques to target desired audiences online to avoid violating social norms, sharing too widely, or sharing with undesired audiences.

\section{Platform-based and ad hoc strategies}

People may, for example, choose online services that provide tools that allow them to target desired audiences, for example choosing to share sensitive content one-on-one using a text messaging tool [34]. They may also use platform-based tools that allow them to target specific portions of their audience. For example, Facebook users may draw on the lists feature to share with certain groups of friends. On email they may share with a specific mailing list [38, 34, 43].

In other cases, the desired audience may be difficult to target using privacy tools explicitly provided by platforms. Users may, instead, draw on ad hoc audience-limiting strategies. They may, for example, control access to their overall Facebook friend networks, self censor, hide or ignore particular friends on their feeds, share on platforms other than Facebook, use multiple Facebook accounts, or develop group norms around the content acceptable to share $[42,19,38,33$, 6, 36, 43].

People may also use audience-reaching strategies, making content widely available and then trying to call desired audience members' attention to it. Litt and Hargittai describe a variety of these techniques including tailoring wording and interacting with audience members through strategies such as tagging and timing posts [22].

\section{Mechanisms for selective sharing}

Facebook currently provides a number of mechanisms to selectively share content, including the ability to share within a friend network, to share with a limited list of people, or to share within public or private groups. However, Facebook provides limited means to share individual pieces of content associated with topics or to search or filter content at the topic level. Facebook also does not allow users to directly opt in or out of viewing content by topic.

Prior work found that Facebook users self censor because of this inability to share with others explicitly interested in individual topics [33]. Thus, users of general sites like Facebook may wish more controls related to topic-level sharing. However, designing these types of controls requires understanding their potential uses as well as the design tensions implicit in adding these types of mechanisms to Facebook, a general-use site. There are a number of topic-based dimensions that could be included in these types of designs.

\section{Tagging}

Services may allow content to be "tagged" with topics so it can be easily searched or filtered $[2,24]$. This mechanism also associates topics with pieces of content rather than the individuals sharing. For example, a user who joins a group related to dogs to share content about dogs identifies themselves as interested in dogs. However, a user doesn't need 
to identify with an interest to share a piece of content tagged as related to dogs. Examples of topic-based tagging mechanisms include the ability to add hashtags on Twitter or the tagging functionalities provided by many blogging or photo sharing platforms.

\section{Opt-in and opt-out mechanisms}

Services may also allow audiences to opt in or out of viewing content by topic. At a high level, users can opt in or out of viewing certain topics on discussion boards by opting to view the forum or not. Similarly, users can decide whether or not to join mailing lists on particular topics.

We expand on prior work by focusing on how audiencereaching and limiting techniques are currently used to target topic-driven audiences across services. We focus on where this type of targeting may fall short and could be supplemented through explicitly topic-focused mechanisms on Facebook, such as topic-based tagging or allowing audience members to opt in or out of content.

\section{Identifiability and sharing}

Users may also sometimes wish to share on topics in a deidentified manner, for example when they consider topics sensitive $[4,15]$. They may also sometimes seek to share in a deidentified manner if content runs counter to how they wish to self-present, for example when sharing about health-related behaviors that may be perceived negatively [27].

Services may allow users to share de-identified content by providing different degrees of anonymity or pseudonymity when sharing on topics. For example, some discussion forums, such as Reddit, are primarily pseudonymous, unlike services such as Facebook that require real names [35]. Other services, such as Twitter, allow for mixed real name and pseudonymous use according to user preference.

Increasing the ability to share in a de-identified or anonymous manner can have both benefits and downsides. Increased anonymity can allow users to share with less inhibition, which can make them more comfortable expressing potentially controversial content [35, 15, 4]. However, designing for this dynamic has inherent tensions. Increased anonymity can also lead to "socially undesirable activities" or decreased civility [15]. Similarly, the use of anonymous sharing can make content appear untrustworthy, if anonymity is not the norm for a platform [35].

Users may wish to share on sensitive topics within an existing network of social media friends, so we examine the possibility of adding mechanisms to facilitate de-identified sharing among Facebook friends. We focus on identifying perceived use cases for de-identified sharing in this network along topicbased dimensions, as well as the tensions around allowing decreased identifiability.

\section{METHODS}

We explored topic-based sharing by first using an interview (n $=16$ ), grounded around a retrospective diary, to examine how people share based on topics across different online services. We then explored the potential impact of adding topic-based sharing to Facebook by asking about the hypothetical impact

\begin{tabular}{ccclc} 
Code & Gender & Age & Occupation & FB Friends \\
\hline P01 & F & 53 & Art/writing & $101-500$ \\
P02 & F & 21 & Student (History, Political Science) & $501-1000$ \\
P03 & F & 20 & Student (Cognitive Science) & $501-1000$ \\
P04 & F & 26 & Scientist/engineer & $101-500$ \\
P05 & F & 26 & Unemployed & $101-500$ \\
P06 & M & 23 & Unemployed & $501-1000$ \\
P07 & M & 27 & Business/management/finance & $101-500$ \\
P08 & F & 26 & PhD Student & $501-1000$ \\
P09 & F & 50 & Administrative support & $501-1000$ \\
P10 & F & 25 & Education & $501-1000$ \\
P11 & M & 30 & Unemployed & $101-500$ \\
P12 & F & 25 & Student (User experience) & $501-1000$ \\
P13 & F & 33 & Unemployed & $501-1000$ \\
P14 & F & 65 & Retired & $51-100$ \\
P15 & M & 23 & Student (Information Science) & $101-500$ \\
P16 & F & 63 & Service & $101-500$
\end{tabular}

Table 1. Participant demographics: participant code, self-reported gender, age, occupational category, number of Facebook friends (range)

of a general topic-based sharing mechanism for each topic. We then used three mockup workflows to probe perceptions of allowing users to: tag content with a topic and then allow their audiences to opt out of viewing it, require their audiences to opt in to see content on a topic, and share on a topic without identifying themselves (de-identified sharing).

\section{Recruitment}

We recruited participants from the Pittsburgh, Pennsylvania area using a Craigslist ad, flyers, and an ad on a Carnegie Mellon recruitment board. Each ad provided a link to a recruitment survey. We sampled for a variety of ages and occupations. We also required participants be over 18 and self-report as highly proficient in English and relatively active on Facebook (defined as having logged into their Facebook account in the past week and posting, on average, at least weekly). Participants were compensated with a \$40 Amazon gift card. Participant demographics are in Table 1.

\section{Retrospective diary}

To prompt discussion of topics around which participants shared, approximately two days before the interview, participants completed retrospective diaries of their online sharing activities. They were sent a link to a survey that asked them to report all the online services they used in the previous week to share content online for personal reasons. For each service, we asked them to open the service and use it to list and describe all the content they shared.

\section{Interview}

Next, we asked participants to complete an approximately hour-long, semi-structured interview in a lab at Carnegie Mellon. One interviewer performed all the interviews, which were audio recorded and transcribed. The interview was intended to: 1) examine topics around which the participant shared across services; 2) explore hypothetical impacts of general topic-based sharing on Facebook; and 3) examine hypothetical use cases and design tensions for three, specific mocked-up topic-based sharing mechanisms on Facebook.

\section{Topic elicitation}

The initial portion of the interview explored topics around which the participant shared online across services. Prior 
work examined general sharing behaviors [33, 22], but did not focus on topic-specific aspects. Our goal was to elicit current topic-based sharing behaviors to understand shortfalls as well as how they compare to more general sharing behaviors.

The interviewer asked the participant to list the topics they typically shared about on the services reported in the diary. The participant was provided with a copy of their retrospective diary to prompt recall of recently shared topics.

The interviewer began by asking the participant to describe ten topics they typically shared about on Facebook. If the participant was not able to list ten, or wanted to identify more, they were encouraged to share the number they felt was appropriate. Participants were told to use their diaries as reference; however, they were told that they could include topics that they had not shared about in the previous week.

After the participant described the topics they typically shared on Facebook, the interviewer went through each topic and asked the participant to:

- Describe the content in more detail (e.g., "What kinds of things do you share related to that?")

- Describe who could see the content (e.g., "Who do you share that with?" "Is that friends only or public?")

- Describe the intended/active audience (e.g., "Is there anyone you particularly want to view it?" "Is there anyone you don't want to view that?" "Who do you think views that?")

- Rate how happy or unhappy they were with who currently viewed the content on a five-point scale from very unhappy to very happy, and explain why

The interviewer repeated this topic elicitation with each service the participant described in their diary. For each service, the interviewer began with the topics the participant had previously described sharing. The interviewer then asked about any additional topics for the service.

After the topic elicitations, the interviewer probed whether the participant considered any of the topics they mentioned sensitive and asked the participant about any topics they tended not to share about online.

\section{General topic-based sharing on Facebook}

The next portion of the interview explored how the participant perceived general, hypothetical topic-based sharing for Facebook for topics currently shared about online, and for topics that they currently chose not to share about online.

The interviewer asked the participant to imagine that they could "post on Facebook for just [their] friends interested in the topic." For this portion, no specific mechanism was provided, to prompt the participant to focus on use cases rather than on design tensions associated with specific mechanisms.

For each topic the participant described sharing about on Facebook, the interviewer asked the participant to rate, on a five-point scale, whether their mood would be happier, unhappier, or the same if they could post content on the topic on Facebook to be seen only by friends interested in the topic.
Next, for each topic they described sharing about on other services, or not sharing about online, the interviewer asked them to rate whether they would be less likely, the same, or more likely to post it on Facebook instead if the content would be shared only with friends interested in the topic.

The interviewer followed up with probing questions about the participant's feelings and who they thought would or would not be interested in each topic. If the participant indicated that they might want to use topic-based sharing, the interviewer probed about potential changes in audience or posting.

Mockups: opt-in, opt-out, de-identified topics

In the next part of the interview the interviewer probed perceived use cases for more concrete versions of topic-based sharing for Facebook. The interviewer presented participants with mockups of three topic-based sharing mechanisms. The mockups were created in Balsamiq and were presented to participants as walkthroughs of hypothetical scenarios. We chose to present the mockups using scenarios to try to prompt reactions to the topic-based interactions, rather than just nuances of the designs.

We created the mockups and chose mechanisms through an iterative process with five pilot participants. We drew on related work for an initial set of requirements that motivated the mechanisms, including the ability to make topics discoverable, allow audiences to search and filter topics, and control identifiability. The mechanisms we chose matched these requirements and met use cases described in piloting.

Each mocked-up mechanism allowed users to "tag" a status update with a topic, in a manner similar to adding a location tag in the Facebook interface current at the time of the study (Feb.-March 2016). In the hypothetical scenario the interviewer asked participants to imagine that they wanted to "share content related to food" with friends interested in food. We chose "food" as a purposefully neutral example, based on pilot responses, to avoid biasing participants toward more extreme use cases. The three scenarios were:

- Opt-out topic-based sharing: For the first workflow the interviewer told the participant to imagine that after tagging the post with "food" they could share it with all their friends. A hypothetical friend could then go to a "topics page," see that they were viewing food content from their friends, and decide to stop viewing food content either for specific friends or for all their friends (Figure 1).

- Opt-in topic-based sharing: In the second workflow the interviewer told the participant to imagine that they didn't want to share with anyone unless an audience member said they wanted to view the topic. In this workflow the participant went to their "topics page," which they could set to require people to opt in to the content. (In the example, the default was opt-out.) They could also decide whether or not to notify people they were sharing on the topic. The workflow user decided to make the topic opt in and not notify anyone. They then posted the food content, and the workflow demonstrated how a hypothetical friend would need to go to their topics page, and opt in to viewing the topic, before the post would appear on their NewsFeed. 


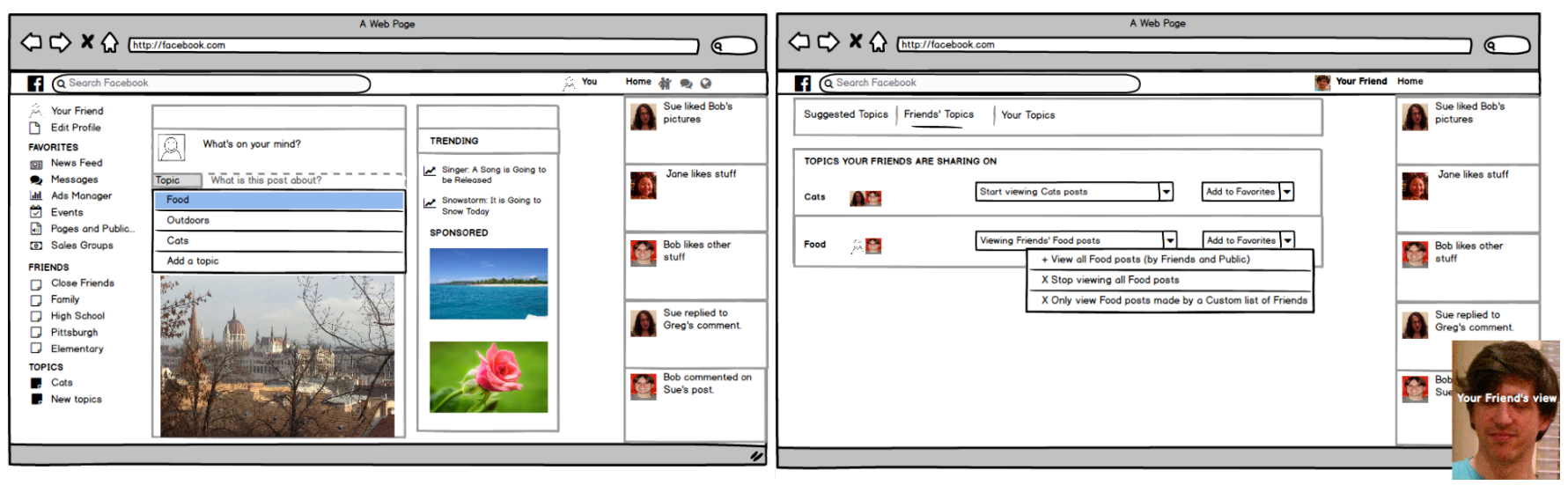

Figure 1. Participants were presented with mocked-up versions of potential topic-based sharing mechanisms created in Balsamiq. The mockups were presented as a series of screens in a scenario. ${ }^{2}$ Two screens from the opt-out scenario are presented above. Participants were told they would tag a post with the topic, which in the example is "food." Then, from their friend's perspective, the content would appear tagged as related to food (not shown here). Their friend would then be able to view their "topics page" and choose if they wanted to continue viewing this food-related content, or not. The full sets of mockup screens are included in the supplementary online materials for this paper.

- De-identified topic-based sharing: In the final workflow the interviewer told the participant to imagine that they could perform opt-in, topic-based sharing but also had the option to check a box to de-identify their posts. The posts would appear on a friend's NewsFeed as posted by "a friend" rather than coming from the participant. In the sample workflow the user checked this box for content related to food, their friend opted in to see food, and the content appeared on their NewsFeed from "a friend."

After each workflow the interviewer asked the participant for general reactions and asked probing questions based on the topics the participants had previously described.

\section{Analysis}

We qualitatively coded the interviews. We created an initial codebook that drew from related work on motivation for use of online services [13, 34, 31, 39], social-networking-site privacy and self-presentation risks [18, 32, 26, 11, 5, 7, 23], sharing and audience-targeting strategies [22, 38], types of online audiences $[16,33,34]$, and the benefits and risks of online anonymity $[35,15,28]$. Two researchers updated this codebook by affinity diagramming three interviews. One researcher then coded the remaining interviews, iteratively updating the codebook.

The final codebook included several high-level categories of codes, which are discussed in this paper, including types of audiences currently targeted through topic-based means (Table 2), current methods for targeting topic-based content, possible benefits of topic-based sharing on Facebook (Table 3), and possible risks topic-based sharing on Facebook could address. We also coded reactions to the specific topic-based sharing mechanisms, including feedback on potential downsides of topic-based sharing on Facebook and perceived engagement strategies. We include some participant counts for

\footnotetext{
${ }^{2}$ Images included in the mockup were accessed through Flickr and are licensed under a creative commons license (https: //creativecommons.org/licenses/by-sa/2.0/legalcode): Flower by Sunny M5, IMG_4728 by Tom Trelvik
}

high-level codes. These are illustrative and are not intended to indicate quantitative significance.

\section{RESULTS}

Participants reported trying to share with audiences interested in specific topics on a range of services. They currently target content along topic-based dimensions through sometimes-broad audience-reaching and limiting strategies, similar to those seen in prior work $[38,22]$. These included a mix of access-control tools, channel choice, sharing content in a generalized format, and self-censoring.

Most participants identified topics for which they thought being able to use topic-based targeting on Facebook rather than broader audience-reaching or limiting strategies on Facebook or other platforms, might allow them to better reach desired audiences. They tended to feel that topic-based targeting might allow them to improve their audiences, share more, improve discussions, or not bother uninterested parties. However, they also described use cases for which they felt topicbased sharing on Facebook would not be sufficient or might be detrimental to their goals, and they outlined different potential strategies for engaging with topic-based sharing mechanisms that would need to be considered for effective design.

\section{Current topic-based sharing across services}

Participants currently draw on ad hoc and platform-supported targeting techniques to try to target audiences based on different types of interests or topics, across Facebook and other services.

\section{Current topic-driven audiences}

Prior work found that social media users tend to want to share with audiences interested in a variety of topics, although available mechanisms may not always facilitate sharing with these types of audiences [14, 33]. Participants described currently intending content they shared, across Facebook and other services, for four primary categories of audiences interested in topics (Table 2), each of which might be facilitated by different types of targeting mechanisms. 


\begin{tabular}{l|l|c}
$\begin{array}{l}\text { Type of interest-based au- } \\
\text { dience (People who...) }\end{array}$ & $\begin{array}{l}\text { Sample topics (sample intended } \\
\text { audiences) }\end{array}$ & \#Part. \\
\hline $\begin{array}{l}\text {..shared traits or life expe- } \\
\text { rience with the participant }\end{array}$ & $\begin{array}{l}\text { university topics (fellow alumni), } \\
\text { museum work (museum profes- } \\
\text { sionals), female to male transition } \\
\text { support (people transitioning), de- } \\
\text { mographic traits (Australians) }\end{array}$ & 13 \\
$\ldots$. would find the topic in- \\
teresting & $\begin{array}{l}\text { cats (people who like cats), TV } \\
\text { shows (show fans), articles related } \\
\text { to innovation (daughter interested } \\
\text { in innovation) }\end{array}$ & 12 \\
...are involved in an event, \\
activity, organization & $\begin{array}{l}\text { organization events (organization } \\
\text { members), parties (invitees), } \\
\text { nights out (other people who were } \\
\text { present), assignments (collabora- } \\
\text { tors on the assignment) } \\
\text { politics (conservatives or liberals), } \\
\text { US presidential election (Hillary } \\
\text { Clinton or Bernie Sanders support- } \\
\text { ers), atheism (religious people) }\end{array}$ & 5 \\
\hline tudes or opinions & 5
\end{tabular}

Table 2. Participants intended content on some topics for audiences with four broad types of interests in the topics. The table includes a description of the types of audiences, sample topics and audiences participants described, and the number of participants who described that type of audience for at least one topic.

Participants intended some topics for audiences they felt would broadly find topics interesting. These topics included content about animals, funny photos, feminist content, or recipes. P02 shared TV-related content, for example, and intended it for "the people that like the fandom."

They also intended content for audiences with certain general attitudes or opinions. This was common for topics related to politics or religion. These participants tended to want to share on these topics with people who held views in line with their own. For example, P09 shared memes that made fun of Donald Trump and intended them for liberal friends. She explained, "I would prefer, actually, that some of the more conservative people weren't looking at them, because I'm not trying to make fun of them personally."

Participants also sometimes intended content for audience members who shared traits or life experiences with them that might prompt interest. Some participants, for example, intended animal-related content for other pet owners. P09 explained that she intended content about her dog for: "other Airedale terrier owners...I don't actually know a lot of my friends, but they're...Airedale terrier owners."

Similarly, some participants wanted to share content related to school or occupations with fellow alumni, classmates, or people with similar professions, such as "people I did teaching with in college" (P10). These participants tended to assume these audiences were interested in related topics.

Finally, some participants intended content for others involved in events, activities, or organizations with them who would share interests in related content. They shared content related to party planning, photos after going out at night, to collaborate when planning an event, or to discuss content related to an organization.

\section{Techniques to target topic-driven content}

In some cases, services provided access-control tools that allowed participants to target topic-driven audiences. In other cases, participants lacked formal mechanisms to target desired topic-based audiences and instead drew on more ad hoc audience-reaching or limiting strategies, reflecting behaviors seen in prior work [38, 22].

For some topics participants drew on formal mechanisms and boundaries to explicitly define and target groups they felt would be interested in the topics. For example, participants used privacy settings, or set up groups of contacts for text messaging or Snapchat. In some cases participants performed this targeting more broadly, at the channel level, by posting on services where they knew that certain audiences would be present who they believed would be interested in a topic.

For other topics, participants chose to only post content on the topic that was acceptable to all audiences on a service, rather than limiting who could view the content. They sometimes did this for all content shared on a platform, reflecting dynamics seen for broader social media sharing [38, 22]. In other cases, they limited the type of content they shared on a specific topic to make it acceptable to a general audience. A few participants, for example, tried to only post objective political content. P12 explained: "I don't really post my personal opinion 'cause my friends...they're not all like me...some might like Trump, some might not, so I just share some articles I've seen on the Internet."

Some participants similarly tried to broadly target topic-based content by controlling overall access [38] to their networks on different services. Several described controlling friend networks on Facebook or other services, so they could freely assume all audience members were willing to view content they posted on different topics. For example P10 explained that, on Twitter, "people that I let follow me are generally people my age range so we'd have similar interests."

Participants also sometimes shared broadly while simultaneously trying to reach some audience members they thought might be interested in particular topics, reflecting some of the "audience-reaching" techniques seen in social media users [22]. Many participants, for example, shared broadly on topics, and assumed only audience members who were interested would "self select" and look at the content. For example, P13 shared content related to feminist topics. She knows she has some friends that "are feminists and they like those all the time...it's not like a private interaction, but not everybody, like, read those, right."

Many participants also shared broadly on topics and then explicitly "interacted" [22] with people they thought might be interested. Participants sometimes shared and then told people about the content, or used tagging, hashtagging, or wall posts. Similarly, a few participants posted broadly but changed the "script" of the post so the meaning would only be clear to relevant audience members [22]. For example, P06 sometimes posted on Facebook about a friend who had passed away. He worded the posts vaguely so that "the people viewing it are either people who know that I'm having 


\begin{tabular}{l|l|l}
$\begin{array}{l}\text { Potential benefit of topic- } \\
\text { based sharing }\end{array}$ & Examples & \#Part. \\
\hline $\begin{array}{l}\text { Post more or better quality } \\
\text { content }\end{array}$ & $\begin{array}{l}\text { Cater basketball-related content to- } \\
\text { ward people who are interested in } \\
\text { basketball } \\
\text { Share with narrower/better } \\
\text { quality audiences }\end{array}$ & $\begin{array}{l}\text { Share pictures of grandchildren } \\
\text { with people who it wouldn't bother }\end{array}$ \\
$\begin{array}{l}\text { Locate people interested in } \\
\text { a topic }\end{array}$ & $\begin{array}{l}\text { Get assistance or social support } \\
\text { (e.g., ask a technical question, ex- } \\
\text { press grief and get support) }\end{array}$ & 8 \\
$\begin{array}{l}\text { Improve discussions on a } \\
\text { topic }\end{array}$ & $\begin{array}{l}\text { Have a more constructive political } \\
\text { conversation, create a "little teacher } \\
\text { forum" for classroom ideas }\end{array}$ & 5
\end{tabular}

Table 3. Participants felt topic-based sharing on Facebook could potentially both reduce the risk of oversharing or annoying/offending others, as well as provide several related benefits. This table describes these potential use case, examples provided by participants, and number of participants who described each benefit.

trouble with her loss, or just like people who think it's just like some vague poetic thing."

\section{Use cases for topic-based sharing on Facebook}

Participants tended to draw on broad audience-reaching or ad hoc techniques to target topic-driven audiences. These strategies sometimes resulted in self-censorship or in the risk of oversharing, for example by not sharing potentially controversial content or assuming others would self-select.

In this section we, therefore, examine how participants felt they might be impacted by an option to share in an explicitly topic-based manner on Facebook. We find that almost all participants felt that they would benefit from topic-based sharing on Facebook for some topics, as a supplement or substitute for current targeting techniques on Facebook and other services. Participants tended to feel that they could use topicbased mechanisms to reduce the risk of boring or annoying audiences as well as to potentially reduce the risk of causing offense. Participants saw a number of potential related benefits, including the ability to target better audiences, find people interested in topics, and post more or better quality content (Table 3). At the same time, adding topic-based mechanisms to Facebook would generate tensions. Topic-based sharing might limit desired reach and, alone, might not meet all sharing needs.

\section{Reducing the risk of overposting or offense}

Several participants felt topic-based sharing on Facebook might reduce the risk of oversharing and of boring or annoying others by posting too much on topics. Participants tended to be concerned about this dynamic for topics they felt might not be interesting to general audiences. These ranged from cat-related posts or pictures of grandchildren to posts about the participant's university; P02 explained, "I have family members that didn't go to [anonymized university] and...they're not close family members so they probably don't care that much...part of the reason I don't post very often on Facebook is that I try not to be annoying."

Some participants also felt targeting interested audiences might help avoid the risk of offending others. This tended to occur for political topics, but also for topics participants felt some audience members might misperceive. P09 described, for example, not sharing on "subculture" topics on Facebook, such as the occult, because she assumed these topics would make people uncomfortable. She would be happier if she could target people interested in countercultural content because it would allow her "to be able to say...'This is my tribe. These are my people. You will understand everything that I'm posting and you won't be offended by it."'

\section{Potential impacts of topic-based sharing}

Participants thought topic-based sharing on Facebook might provide benefits along with reducing these risks (Table 3).

Some participants felt topic-driven sharing might allow them to specifically post to narrower, interested, better-quality, audiences, rather than posting broadly and relying on audiences to self-select. Perceptions of these improved audiences varied. Participants sometimes wanted audiences filtered for people who wouldn't be offended or annoyed. P01 envisioned sharing pictures of her grandchildren with: "A grandma and close friends list that I could send those to, and not bother the rest of the group with."

Some participants also felt that topic-based mechanisms would facilitate locating people interested in topics. Some felt that targeting content on a topic at people interested in that topic might generally increase the attention paid to posts. Similarly, participants sometimes felt targeting people interested in a topic might lead to more effective assistance or social support. In one case, P12 described using Facebook to ask technical questions. She felt that narrowing the audience to people interested in technical topics would increase the likelihood of useful help: "I think people would be more interested in helping me because...they're closer to me."

Several participants felt that targeting more focused audiences might allow for improved discussions on topics. This dynamic occurred for political content for which participants thought limiting the audience to people interested might lead to a more constructive or informed conversations. However, participants also felt that it might prompt better discussion in other areas. For example, P10, a teacher, liked the idea of using topic-based sharing to post on Facebook about classroom design ideas she currently posts about on Pinterest: "It would be almost like a little teacher forum...'cause it's always nice to bounce ideas off each other."

Similarly, a few participants felt that if they knew they were sharing for an interested audience they might post more, more targeted, or better quality, content. P11 posted basketballrelated content, and explained that if he were posting for people interested in basketball he could "sort of cater it towards the people that are interested in it, who want to see it."

\section{Potential uses of de-identified topics on Facebook}

Using our mockups we also probed perceived uses and tensions for sharing on topics in a de-identified manner on Facebook. Prior work has found tensions between the desire to share sensitive content in a de-identified manner, and the potential abuse of de-identified sharing [15, 28, 4]. 
Similarly, most participants perceived de-identified sharing negatively. Some felt, in line with prior work $[15,28]$, that sharing without a tie to one's identity on Facebook would be creepy, suspicious, or inappropriate for the platform. However, two participants pointed out use cases that illustrate how de-identified sharing might be useful for some sensitive topics. P06, who is transgender, explained that de-identified sharing might be useful for support during the transition process for sensitive or embarrassing questions. He explained, for example, "one of the, like, side effects of hormones is acne. And so like I don't necessarily want people to know that I'm posting about it, but it would be nice to like write about it, and then get some responses like, 'Oh, this is what I tried."'

Similarly, P11 had previously worked as a police officer. He explained that it would be useful to be able to post on topics in a de-identified manner to respond to negative statements about police: "Some people post some pretty nasty stuff, um, so I guess being able to put my point of view forward... 'cause you can get in trouble for certain things."

\section{Design tensions for topic-based sharing}

Tools designed to facilitate topic-based sharing would also need to consider where topic-based mechanisms on a generaluse site such as Facebook might have negative impacts or fall short. All participants also described topics for which topicbased sharing mechanisms on Facebook would not improve their ability to share, or, if used, could be detrimental.

\section{Explicit privacy needs}

Some participants described topics for which they wanted explicit privacy controls. These topics included pictures with other people, personal topics, or funny things they felt might be embarrassing if broadcast. In these cases, they tended to feel that topic-based sharing mechanisms on Facebook would be insufficient, because they wouldn't provide additional explicit privacy protections. Instead, they tended to want to share privacy-sensitive topics on services that allowed them to explicitly limit audiences, or, in some cases, didn't want to share on these topics online. They were concerned about protecting their own or others' privacy, and, in a few cases, protecting the content from Facebook itself.

\section{Peripheral audiences and stratification}

Users sometimes broadcast on Facebook to reach "peripheral audiences" beyond those the user may specifically consider [22]. A few participants described topics for which they wanted to reach audiences who might be prompted to become interested in the topics, rather than use topic-based sharing tools to target people already interested. For these topics they felt using topic-focused sharing would be counter-productive. For example, P03 described using Facebook for a fundraiser: "We marketed it just to the people we knew in like our community, but we ended up making $\$ 700.00$ in sales just from this other organization that, you know, one person saw it, shared it to their group or something...And that's kind of a situation where we didn't even know they were interested." She felt topic-based targeting would not be useful for this type of sharing.
Participants also brought up the possibility that topicbased sharing might contributing to undesirable "filter bubbles" [12]. A few participants felt topic-based sharing might limit the diversity of viewpoints they would be able to expose their friends to, increasing undesirable dynamics such as political stratification. P15, for example, wouldn't want to use topic-based sharing for atheism-related posts, because "the other people don't get to see alternative viewpoints, and I think that's important.”

\section{Topic creation and management}

We presented participants with mockups in which topic-based sharing was performed by manually tagging posts with topics and then viewing one's own topics and friends' topics on a "topics page" (modeled off the page used to manage Facebook Groups in the current interface). Participants described a wide variety of topics on which they currently shared that would need to be added, ranging from "politics" or "dinosaurs" to more contextually-defined topics such as "funny" or "personal."

Several participants were concerned that it might take too much time to add these types of topics to posts, or that long lists of topics might become difficult to manage. A topicdriven sharing design that depended on tagging posts with topics would need to consider how to make these processes fast and usable.

\section{Strategies for topic-based opt-in and opt-out}

Designers would also need to consider how users might want to engage with topic-based sharing and viewing mechanisms on Facebook, a general-use site. Participants described highlevel strategies they felt would shape use of topic-based opt-in and opt-out mechanisms.

Relationship-driven decisions versus content-driven decisions Participants tended to describe different high-level strategies for deciding whether to view friends' content by topic, when presented with the opt-in and opt-out design mockups. Some strategies tended to be primarily relationship-driven, while others were more content-driven.

Several participants felt that they would decide whether to view content based on the person sharing, rather than the topic. For example, P15 explained, "I don't think I filter content based on what it's about, but more related to who's posting it...I attribute more credibility to people rather than the things that are being posted." Some of these participants didn't like the idea of choosing topics to view using opt-in or opt-out mechanisms. As P14 described: "I want to see what my friends are interested in...And if that means that I'm seeing things about them that I may not like, well, so-so be it. They are who they are."

Rather than opting out of content by topic, these participants sometimes felt they would prefer to unfriend or unfollow someone if they met a threshold of objectionable posts. Other participants who prioritized viewing content on the individual level felt that they might want to opt in or out of some topics but would choose to do so on a per-friend basis. 
Other participants, however, liked the idea of being able to adjust content they viewed by turning topics on and off across friends, rather than focusing on viewing posts on a friend-byfriend basis. Some felt content shared on some topics was overwhelming: "You want people to remain your friends per se, but you don't necessarily want to see all the, oh, she's posting about her running a marathon again" (P09).

These participants also sometimes felt that filtering topics might let them remain friends with people who shared some material they found offensive or annoying: "Some people post things that I find very inflammatory and uninformed and I tend to want to argue with them. And if I don't see [those posts], then I don't want to argue with them, and then I can still be friends with them" (P05).

\section{Opt-in versus opt-out sharing}

Using the mockups we also explored how participants perceived sharing using opt-in versus opt-out by topic. In most cases participants preferred the idea of allowing others to opt out of content. Several participants, for example, felt that optin-based mechanisms might require too much work to view the content. Some also felt that opt-in might remove the ability to discover unexpected content, which they felt was a benefit of Facebook.

However, participants described several sharing strategies that would benefit from opt-in mechanisms that should also be considered when designing topic-driven mechanisms. A few participants described topics they'd like to share for which they'd want to know that their audiences had explicitly expressed interest, and for which opt-in might be useful. P04 explained, for example, that she might want to use opt-in for sharing about television shows: "You would assume that people wouldn't be angry about Game of Throne spoilers if they were opted in to, like, read posts about it."

\section{LIMITATIONS}

This work is intended to be exploratory. We chose an interview-based approach to establish deeper insights into topic-based sharing. We drew on diaries and included mockups to ground the interviews in participants' sharing activities and to help participants understand the context. We chose to focus on Facebook because it allowed us to discuss concrete sharing mechanisms with participants. It also provided a well-studied platform that permitted us to ground our findings in established understandings of broader uses, benefits, audiences, and sharing strategies and challenges.

These methodological choices, however, have tradeoffs. Interview studies are limited in scope and generalizability. Our sample, for example, trended female and relatively young, and it included a relatively high percentage of students and unemployed participants. Using mockups may also have biased participants by presenting explicit scenarios, and focusing on Facebook limited insights to a single platform. Furthermore, responses about hypothetical uses may not reflect actual use patterns. However, we felt these tradeoffs were appropriate for our goals. In piloting we found that discussing hypothetical sharing mechanisms without examples or concrete context tended to confuse participants. We chose, therefore, to use interviews and to include Facebook-based mockup workflows to provide concrete examples of the sharing mechanisms we wanted to discuss. Future work could address these limits by using fuller implementations of topicbased mechanisms to provide direct insights about usability. Additional studies could also use survey-based methods to focus on generalizing findings across different types of users or platforms.

This work used a diary-driven approach and recruited from a general population. We, therefore, primarily focused on everyday topics (e.g., politics, animals, hobbies, etc) and related use cases. However, users may also benefit from topicbased sharing in more niche, potentially sensitive situations such as health-related sharing or crisis management. Healthrelated sharing, for example, has been found to benefit from granular access controls $[10,30]$ and provides examples of topics users may hesitate to share about broadly due to potential negative perceptions, despite potential benefits [9]. These types of topics may not emerge naturally when looking at the general population's everyday sharing. Additional work could specifically focus on topic-based sharing needs and challenges for these types of use cases, for example by recruiting populations who may be experiencing health- or crisis-related events.

\section{DISCUSSION}

These findings offer design implications for topic-based sharing mechanisms on Facebook, as well as some insights for other services that seek to support topic-based sharing.

\section{Support for different types of interest-based audiences}

Across platforms participants wanted to target content for several types of interest-driven audiences, for different topics (Table 2). To address these needs, Facebook and other services should focus on allowing users to target a range of interest and topic-driven audiences, which may require different types of sharing mechanisms.

Services currently provide mechanisms to explicitly target these different types of interest-driven audiences to different degrees. Facebook, for example, directly supports finding people involved in events/activities/organizations, through Facebook Groups or Events. It is also sometimes possible to find or create groups or lists associated with a trait or opinion, when that trait or opinion is known or where the trait aligns with an identifiable life stage (e.g., high school friends). However, it is currently relatively difficult on Facebook to find people who generally find topics interesting or who have general attitudes or opinions that they don't identify with on a group level.

When there are gaps in how a service allows users to target specific types of interest-driven audiences, users may fall back on general targeting methods, which may lead to oversharing or self-censorship. Adapting sharing mechanisms to target these gaps on different services for different types of interest-driven audiences may allow users to better target desired audiences for topics. 


\section{Focusing on relationships versus content for Facebook}

We observed that when deciding whether they might want to opt out of viewing content on Facebook some participants felt that they would base viewing decisions on relationships with others, while other participants would prefer to base viewing decisions on topics. To capture both these types of preferences, topic-based sharing tools on Facebook could allow the granularity to make opt-out and opt-in decisions both at the topic and individual levels. For example a tool could include both the ability to opt out of food-related content for all friends or opt out of food-related content for just a specific friend. This granularity might allow users to base decisions on both interests and relationships.

\section{Need to combine topic-based sharing and explicit control} Prior work found that selective sharing, including topic-based targeting, takes place in the context of other tasks and sharing needs [34]. Similarly, we observed that participants found topic-based sharing on Facebook insufficient for some topics because of a desire for more explicit privacy guarantees. Users may want to target particular, topic-driven audiences but may not want to give up the ability to simultaneously limit the overall audience. Future work should explore the impact of combining topic-based sharing and the ability to transparently, and explicitly, limit audiences. This could be done by incorporating privacy guarantees into topic-based targeting on Facebook or by exploring topic-based targeting on services with more explicit privacy controls.

\section{Transparency into viewership}

Some participants also felt that if they knew people were interested in content posted on topics on Facebook they might post more or better content. However, to achieve this benefit, participants also sometimes wanted transparency into whether anyone was interested in a topic, who was interested, or how many people were interested. Topic-based sharing design for Facebook should consider whether and how to inform people about the number of people in the active audience. Prior work has found that impressions of viewership can depend on cues provided by the service as well as user agency [21]. Future work could examine the impact on potential posting behaviors of informing people when or if people opted in or out of viewing their content for various topics.

Topic-based sharing mechanisms for Facebook should also consider how to incorporate notifications for sharing on particular topics. In our mockups the user was able to notify audiences about topics audiences could opt into. Some participants felt that these types of notifications could be bothersome, while others felt that they would prefer to notify their audiences and not depend on them finding the content on their own. A topic-based mechanism could seek to support both types of topic-based sharing-topics that users share about and notify their audience about to give them the option to opt into, and topics the user might want to share about and make available for search without explicit notification.

\section{Facilitating topic management on Facebook}

A topic-driven sharing design would need also to consider how to make adding and organizing topics fast and usable on the Facebook platform.

\section{Automation}

Automating or partially automating the process of adding topic-based tags to content might increase usability by increasing speed and consistency. For example, tags for some topics could be repurposed from existing metadata or organizational uses [17]. For other topics, it might be possible to infer topics from content at the user or post level. However, depending on where data was drawn from and how it was used, inferred topics might be seen as privacy-invasive. Some topics can also be difficult to infer accurately. For example, contextual topics such as "funny" can be difficult to tag automatically [8], while it might be easier to automatically infer non-contextual topics such as "dinosaur." Future work could examine the tradeoffs among inferred tags, usercreated tags, and/or combined systems. Evaluation could focus on factors including speed, privacy concerns, accuracy and consistency, and usefulness for achieving the benefits described in this work.

Providing tools to help visualize and manage tags could also facilitate this process. For example, prompting users to begin with a pre-selected list of tags could help maintain a manageable number of tags, Similarly, providing an easy way to view, sort, and edit all tags in bulk could allow users to organize and manage their topics.

\section{Other patterns for grouping content or users by topic}

Facebook could also draw on design patterns other services use for topic-based organization and sharing, beyond explicit tagging. Examples include allowing users to create or post to topic-specific forums or feeds, or allowing friends to sign up for topic-based mailing lists. Future work could explore whether these types of mechanisms might increase usability, in the Facebook context, for the topic-based use cases seen in this study.

\section{CONCLUSION}

We found that adding topic-based sharing on Facebook has the potential to allow people to better share content with their desired audiences. There were a number of use cases for Facebook-based topic-oriented sharing including reducing the risk of oversharing or causing offense, sharing with a narrower or better audience, improving discussions, allowing participants to locate interested audiences, and potentially allowing participants to share better content with a more targeted audience. To create usable topic-based sharing mechanisms for Facebook, however, designers would need to consider needs unmet by topic-based sharing alone, as well as use cases for potential engagement strategies.

\section{ACKNOWLEDGMENTS}

This material is based upon work supported by the National Science Foundation under Grants No. 0946825 and CNS1012763, as well as the ARCS Foundation. Thank you to Lujo Bauer, Moira Burke, and Laura Dabbish for their contributions. 


\section{REFERENCES}

1. Alessandro Acquisti and Ralph Gross. 2006. Imagined communities: Awareness, information sharing, and privacy on the Facebook. In Privacy Enhancing Technologies. Springer, 36-58. http: //link. springer. com/chapter/10.1007/11957454_3

2. Morgan Ames and Mor Naaman. 2007. Why we tag: Motivations for annotation in mobile and online media. In Proceedings of the SIGCHI Conference on Human Factors in Computing Systems. ACM, 971-980. http://dl.acm.org/citation. cfm?id=1240772

3. Michael S Bernstein, Eytan Bakshy, Moira Burke, and Brian Karrer. 2013. Quantifying the invisible audience in social networks. In Proceedings of the SIGCHI Conference on Human Factors in Computing Systems. ACM, 21-30.

http: //dl . acm. org/citation. $\mathrm{cfm}$ ?doid=2470654. 2470658

4. Jeremy Birnholtz, Nicholas Aaron Ross Merola, and Arindam Paul. 2015. Is it weird to sill be a virgin?: Anonymous, locally targeted questions on Facebook Confession Boards. In Proceedings of the ACM Conference on Human Factors in Computing Systems. ACM, 2613-2622. http: //comm. soc . northwestern. edu/ socialmedia/files/2012/09/pn1506-birnholtz .pdf

5. Danah Boyd. 2006. Friends, friendsters, and myspace top 8: Writing community into being on social network sites. (2006). http: //citeseerx . ist. psu. edu/viewdoc/ summary?doi=10.1.1.79.5665

6. Sauvik Das and Adam Kramer. 2013. Self-Censorship on Facebook.. In ICWSM.

http: //www . aaai . org/ocs/index . php/ICWSM/ICWSM13/paper/ viewPDFInterstitial/6093/6350

7. Nicole B. Ellison, Jessica Vitak, Rebecca Gray, and Cliff Lampe. 2014. Cultivating social resources on social network sites: Facebook relationship maintenance behaviors and their role in social capital processes. Journal of Computer-Mediated Communication 19, 4 (2014), 855-870. http:

//onlinelibrary.wiley.com/doi/10.1111/jcc4.12078/full

8. Roberto González-Ibánez, Smaranda Muresan, and Nina Wacholder. 2011. Identifying sarcasm in Twitter: A closer look. In Proceedings of the 49th Annual Meeting of the Association for Computational Linguistics: Human Language Technologies: Short papers-Volume 2. Association for Computational Linguistics, 581-586.

9. Kathryn Greene and S Petronio. 2000. Disclosure of chronic illness varies by topic and target: The role of stigma and boundaries in willingness to disclose. In Balancing the Secrets of Private Disclosures. Lawrence Erlbaum Associates, 123-135.

10. Andrea Hartzler, Meredith M Skeels, Marlee Mukai, Christopher Powell, Predrag Klasnja, and Wanda Pratt. 2011. Sharing is caring, but not error free: Transparency of granular controls for sharing personal health information in social networks. In AMIA Annual
Symposium Proceedings, Vol. 2011. 559-568.

http://pubmedcentralcanada.ca/pmcc/articles/ PMC3243199/pdf/0559_amia_2011_proc.pdf

11. Bernie Hogan. 2010. The presentation of self in the age of social media: Distinguishing performances and exhibitions online. Bulletin of Science, Technology \& Society (2010). http: //bst. sagepub. com/content/early/ 2010/10/31/0270467610385893. abstract

12. The White House. 2014. Big Data: Seizing Opportunities, Preserving Values. (2014). https://www . whitehouse . gov/sites/default/files/docs/ big_data_privacy_report_may_1_2014.pdf

13. Adam N. Joinson. 2008. Looking at, looking up or keeping up with people?: Motives and use of Facebook. In Proceedings of the SIGCHI Conference on Human Factors in Computing Systems. ACM, 1027-1036. http://dl . acm.org/citation. cfm?id=1357213

14. Sanjay Kairam, Mike Brzozowski, David Huffaker, and Ed Chi. 2012. Talking in circles: Selective sharing in Google+. In Proceedings of the SIGCHI Conference on Human Factors in Computing Systems. ACM, 1065-1074. http: //dl . acm. org/citation. cfm?id=2208552

15. Ruogu Kang, Stephanie Brown, and Sara Kiesler. 2013. Why do people seek anonymity on the Internet? Informing policy and design. In Proceedings of the SIGCHI Conference on Human Factors in Computing Systems. ACM, 2657-2666.

http: //dl . acm.org/citation. cfm?id=2481368

16. Patrick Gage Kelley, Robin Brewer, Yael Mayer, Lorrie Faith Cranor, and Norman Sadeh. 2011. An investigation into Facebook friend grouping. In Human-Computer Interaction, INTERACT, 2011. Springer, 216-233. http://link. springer. com/chapter/ 10. 1007/978-3-642-23765-2_15

17. Peter Klemperer, Yuan Liang, Michelle Mazurek, Manya Sleeper, Blase Ur, Lujo Bauer, Lorrie Faith Cranor, Nitin Gupta, and Michael Reiter. 2012. Tag, you can see it!: Using tags for access control in photo sharing. In Proceedings of the SIGCHI Conference on Human Factors in Computing Systems. ACM, 377-386. http : //dl . acm. org/citation. cfm?id=2207728

18. Haewoon Kwak, Hyunwoo Chun, and Sue Moon. 2011. Fragile online relationship: A first look at unfollow dynamics in Twitter. In Proceedings of the SIGCHI Conference on Human Factors in Computing Systems. ACM, 1091-1100.

http: //dl . acm. org/citation. c fm?id=1979104

19. Airi Lampinen, Vilma Lehtinen, Asko Lehmuskallio, and Sakari Tamminen. 2011. We're in it together: Interpersonal management of disclosure in social network services. In Proceedings of the SIGCHI Conference on Human Factors in Computing Systems. ACM, 3217-3226.

http://dl.acm.org/citation. cfm?id=1979420 
20. A. Lampinen, S. Tamminen, and A. Oulasvirta. 2009. All my people right here, right now: Management of group co-presence on a social networking site. In Proceedings of the ACM 2009 International Conference on Supporting Group Work. 281-290.

http://dl. acm.org/citation. cfm?doid=1531674.1531717

21. Eden Litt. 2012. Knock, knock. Who's there? The imagined audience. Journal of Broadcasting \& Electronic Media 56, 3 (2012), 330-345. http://www. tandfonline . com/doi/abs/10.1080/08838151.2012.705195

22. Eden Litt and Eszter Hargittai. 2016. "Just cast the net, and hopefully the right fish swim into it": Audience management on social network sites. In Proceedings of the ACM Conference on Computer-Supported Cooperative Work \& Social Computing. ACM, New York, NY, USA, 1488-1500. DOI :

http://dx.doi .org/10.1145/2818048.2819933

23. Eden Litt, Erin Spottswood, Jeremy Birnholtz, Jeff T. Hancock, Madeline E. Smith, and Lindsay Reynolds. 2014. Awkward encounters of an other kind: Collective self-presentation and face threat on Facebook. In Proceedings of the ACM Conference on Computer Supported Cooperative Work \& Social Computing. ACM, 449-460.

http://dl . acm . org/citation. cfm?id=2531646

24. Cameron Marlow, Mor Naaman, Danah Boyd, and Marc Davis. 2006. HT06, tagging paper, taxonomy, Flickr, academic article, to read. In Proceedings of the Seventeenth Conference on Hypertext and Hypermedia. ACM, 31-40.

http://dl . acm . org/citation. cfm?doid=1149941. 1149949

25. Alice E. Marwick and danah boyd. 2011. I tweet honestly, I tweet passionately: Twitter users, context collapse, and the imagined audience. New Media \& Society 13, 1 (2011), 114-133.

http: //nms . sagepub. com/content/13/1/114 . short

26. Caitlin McLaughlin and Jessica Vitak. 2012. Norm evolution and violation on Facebook. New Media \& Society 14, 2 (March 2012), 299-315. DOI:

http://dx.doi.org/10.1177/1461444811412712

27. Mark W Newman, Debra Lauterbach, Sean A Munson, Paul Resnick, and Margaret E Morris. 2011. It's not that I don't have problems, I'm just not putting them on Facebook: Challenges and opportunities in using online social networks for health. In Proceedings of the ACM Conference on Computer Supported Cooperative Work. ACM, 341-350.

http: //dl . acm . org/citation. cfm?doid=1958824 . 1958876

28. Sai Teja Peddinti, Keith W. Ross, and Justin Cappos. 2014. "On the Internet, nobody knows you're a dog": A Twitter case study of anonymity in social networks. In Proceedings of the Second ACM Conference on Online Social Networks. ACM, New York, NY, USA, 83-94. http://doi . acm.org/10.1145/2660460.2660467

29. Sandra Petronio. 2012. Boundaries of privacy: Dialectics of disclosure. Suny Press.
30. Lillian Røstad and Ole Andreas Alsos. 2009. Patient-administered access control: A usability study. In International Conference on Availability, Reliability and Security International. IEEE, 877-881.

31. Bryan Semaan, Heather Faucett, Scott Robertson, Misa Maruyama, and Sara Douglas. 2015. Navigating Imagined Audiences: Motivations for Participating in the Online Public Sphere. In Proceedings of the ACM Conference on Computer Supported Cooperative Work \& Social Computing. ACM, New York, NY, USA, 1158-1169. DOI :

http://dx. doi . org/10.1145/2675133.2675187

32. Christopher Sibona and Steven Walczak. 2011. Unfriending on Facebook: Friend request and online/offline behavior analysis. In Hawaii International Conference on System Sciences. IEEE, 1-10. http:// ieeexplore . ieee. org/xpls/abs_all . jsp?arnumber $=5718725$

33. Manya Sleeper, Rebecca Balebako, Sauvik Das, Amber Lynn McConahy, Jason Wiese, and Lorrie Faith Cranor. 2013. The post that wasn't: Exploring self-censorship on Facebook. In Proceedings of the ACM Conference on Computer Supported Cooperative Work. ACM, 793-802.

http: //dl . acm. org/citation. cfm?id=2441865

34. Manya Sleeper, William Melicher, Hana Habib, Lujo Bauer, Lorrie Faith Cranor, and Michelle L Mazurek. 2016. Sharing Personal Content Online: Exploring Channel Choice and Multi-Channel Behaviors. In Proceedings of the ACM Conference on Human Factors in Computing Systems. ACM.

http://dl . acm. org/citation. cfm?doid=2858036.2858170

35. H. Colleen Stuart, Laura Dabbish, Sara Kiesler, Peter Kinnaird, and Ruogu Kang. 2012. Social transparency in networked information exchange: A theoretical framework. In Proceedings of the ACM Conference on Computer Supported Cooperative Work. ACM, 451-460. http: //dl . acm.org/citation. . fm?id=2145275

36. Frederic Stutzman and Woodrow Hartzog. 2012. Boundary regulation in social media. In Proceedings of the ACM 2012 Conference on Computer Supported Cooperative Work. ACM, 769-778.

http: //dl . acm. org/citation. cfm?doid=2145204. 2145320

37. Eran Toch, Justin Cranshaw, Paul Hankes Drielsma, Janice Y Tsai, Patrick Gage Kelley, James Springfield, Lorrie Cranor, Jason Hong, and Norman Sadeh. 2010. Empirical models of privacy in location sharing. In Proceedings of the ACM International Conference on Ubiquitous Computing. ACM, 129-138. http://dl . acm. org/citation. cfm?doid=1864349 . 1864364

38. Jessica Vitak, Stacy Blasiola, Sameer Patil, and Eden Litt. 2015. Balancing audience and privacy tensions on social network sites: Strategies of highly engaged users. International Journal of Communication 9 (2015), 20. http://ijoc.org/index.php/ijoc/article/view/3208 
39. Jessica Vitak and Nicole B. Ellison. 2012. “There's a network out there you might as well tap": Exploring the benefits of and barriers to exchanging informational and support-based resources on Facebook. New Media \& Society (2012). http: //nms . sagepub. com/content/early/ 2012/07/17/1461444812451566. abstract

40. Yang Wang, Gregory Norcie, Saranga Komanduri, Alessandro Acquisti, Pedro Giovanni Leon, and Lorrie Faith Cranor. 2011. I regretted the minute I pressed share: A qualitative study of regrets on Facebook. In Proceedings of the Seventh Symposium on Usable Privacy and Security. ACM, 10.

http: //dl . acm. org/citation. cfm?id=2078841

41. Jason Wiese, Patrick Gage Kelley, Lorrie Faith Cranor, Laura Dabbish, Jason I. Hong, and John Zimmerman. 2011. Are you close with me? Are you nearby?: Investigating social groups, closeness, and willingness to share. In Proceedings of the International Conference on Ubiquitous Computing. ACM, New York, NY, USA, 197-206. DOI :

http://dx. doi . org/10.1145/2030112.2030140

42. Pamela Wisniewski, Heather Lipford, and David Wilson. 2012. Fighting for my space: Coping mechanisms for SNS boundary regulation. In Proceedings of the SIGCHI Conference on Human Factors in Computing Systems. ACM, 609-618.

43. Pamela J Wisniewski, Bart P Knijnenburg, and Heather Richter Lipford. 2017. Making privacy personal: Profiling social network users to inform privacy education and nudging. International Journal of Human-Computer Studies 98 (2017), 95-108.

44. Xuan Zhao, Cliff Lampe, and Nicole B Ellison. 2016. The Social Media Ecology: User Perceptions, Strategies and Challenges. In Proceedings of the ACM Conference on Human Factors in Computing Systems. ACM, 89-100. 